\title{
Quantitative Analysis of Protein-Protein Interactions: One- and Two-photon Excitation FRET-FLIM Microscopy
}

\author{
Ammasi Periasamy and Ye Chen \\ W. M. Keck Center for Cellular Imaging, Departments of Biology and Biomedical Engineering, \\ University of Virginia, Gilmer Hall (064), Charlottesville, VA 22904, USA
}

Visualizing and quantifying protein-protein interactions is a recent trend in biomedical imaging. The current advances in fluorescence microscopy coupled with the development of new fluorescent probes and detectors provide the tools to study protein associations in living specimens [1-2]. Förster resonance energy transfer (FRET) occurs when two fluorophores (donor and acceptor) have a sufficiently large spectral overlap, a favorable dipole-dipole orientation, proximity of 1-10 nm and a large enough quantum yield $[1,3]$. Upon energy transfer, donor fluorescence is quenched and acceptor fluorescence is increased (sensitized), resulting in a decrease in donor excitation intensity or lifetime. The fluorophore molecule used for FRET imaging has a characteristic absorption and emission spectrum that should be considered for characterizing the FRET signal acquired using oneand two-photon excitation FRET microscopy [1].

There are a number of methods to avoid, minimize or correct the spectral bleedthrough (SBT) contamination in intensity-based FRET, each having specific limitations depending on the level of sensitivity desired $[1,4]$. We have developed an algorithm to correct the contamination in the FRET image to estimate the energy transfer efficiency (E) and the distance (r) between donor and acceptor molecule $[1,5]$. Our algorithm requires seven images to remove the donor (DSBT) and the acceptor (ASBT) SBT from the contaminated FRET or uncorrected FRET (uFRET) to obtain the processed FRET (PFRET) image as shown in Figure 1. The E\% and the distance (r) between donor and acceptor molecules in GHFT1-5 cell nucleus are considerably changed after SBT correction.

In contrast, fluorescence lifetime imaging (FLIM) is independent of excitation intensity or fluorophore concentration. The combination of FLIM and FRET will provide high-speed, high spatial (nanometer), and temporal (nanoseconds) resolution when compared to one- and two-photon excitation FRET imaging [1,6]. By measuring the donor lifetime in the presence and the absence of acceptor one can accurately calculate the energy transfer efficiency and the distance between donorand acceptor-labeled proteins in living specimens. As shown in Figure 2 protein expressed with ECFP shows a single lifetime distribution contrary to the literature [7]. The ratio of the lifetimes and the pre-exponential factors would help to determine in identifying the percentage of the interacting and non-interacting protein molecules (see Figure 2).

References

[1] A. Periasamy and R.N. Day, Molecular Imaging:FRET Microscopy and Spectroscopy, Oxford University Press, New York, 2005.

[2] H. Wallrabe and A. Periasamy, Curr. Opin. BioTech. 16 (2005) In Press.

[3] T. Förster, Ann. Phys. 2 (1948) 55. 


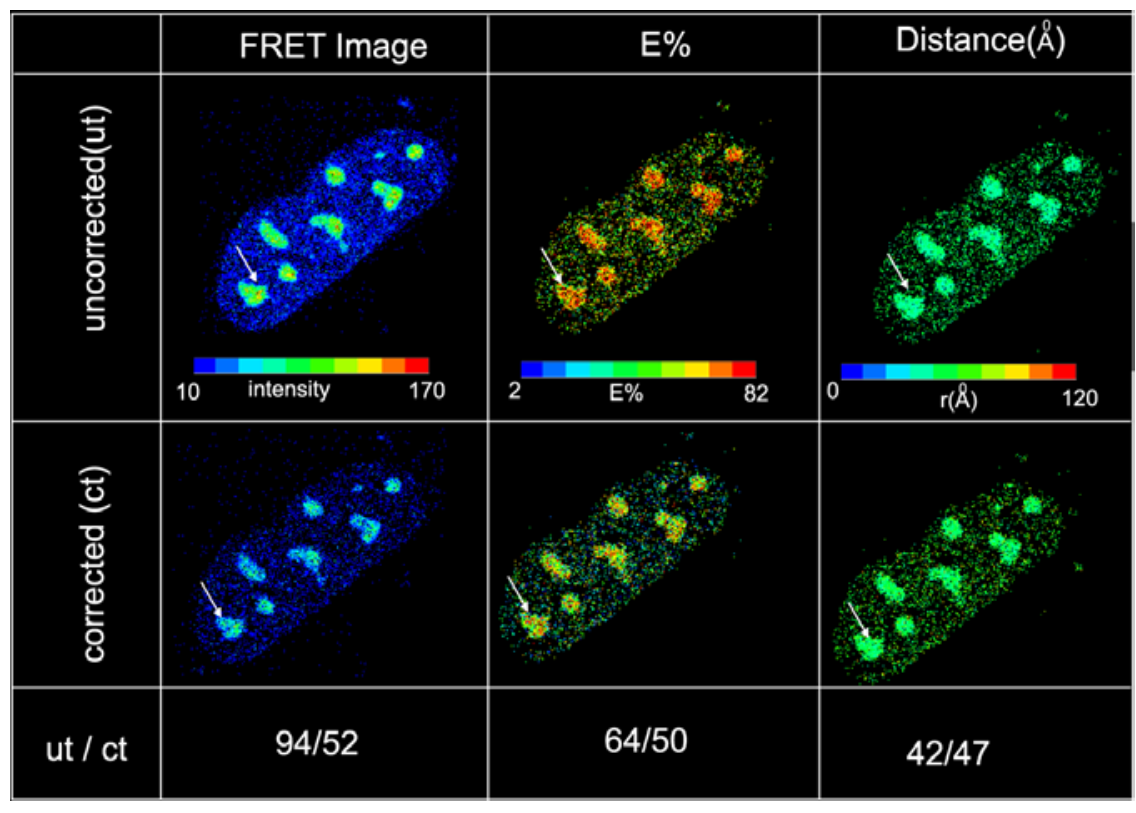

[4] C. Barney and G. Danuser, Biophys. J. 84 (2003) 3992.

[5] M. Elangovan et al., Methods 29 (2003) 58.

[6] R. M. Clegg et al., Methods Enzymol. 360 (2003) 509.

[7] M. A. Rizzo et al., Nature Biotech. 22 (2004) 445. The aid of Dr. R.N. Day is gratefully acknowledged.

Figure 1. Intensity-based quantitative 2p-FRET data analysis to localize the dimerization of $\mathrm{C} / \mathrm{EBP} \alpha$ proteins in GHFT1-5 cell nucleus. Seven images were acquired using Biorad Radiance confocal/multiphoton microscopy for PFRET data analysis [1, 5; www.circusoft.com]. The color bars clearly demonstrate the implementation of ASBT and DSBT correction. The top panel shows the uncorrected FRET, E\%, and distance images and their respective color bars. The bottom panel shows the two-dimensional distribution of corrected FRET, efficiency $(\mathrm{E} \%)$, and the distance (r) images. The rate of energy transfer efficiency (E) is $64 \%$ before correction and 50\% after DSBT and ASBT correction for a

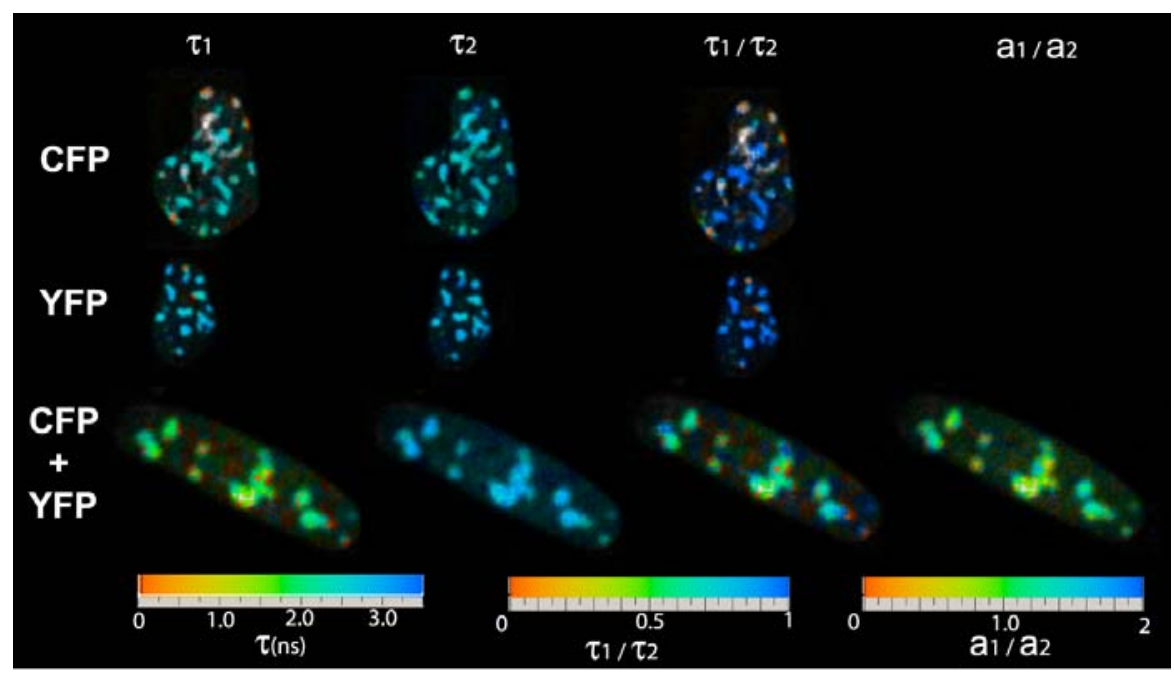
protein complex (arrow).

Figure 2. FLIM based quantitative FRET data analysis. The lifetime of the donor $(\mathrm{CFP}-\mathrm{C} / \mathrm{EBP} \alpha)$ molecule was measured in the absence (mean $\tau_{\mathrm{D}}=2.5 \mathrm{~ns}$ ) and in the presence of acceptor (mean $\left.\tau_{\mathrm{DA}}=1.5 \mathrm{~ns}\right)$. The CFP alone expressed proteins clearly shows single component lifetime distribution in the $\tau_{1} / \tau_{2}=1$ for two component analysis. On the other hand the $\tau_{1} / \tau_{2}$ ratio distribution from 0 to 1 in the presence of acceptor molecules. This clearly shows double exponential decay indicating the occurrence of protein dimerization in the GHFT1-5 cell nucleus. The preexponential factor $\mathrm{a}_{1} / \mathrm{a}_{2}$ also shows clear relationship with the lifetime distribution of the donor and acceptor molecules. But in the case of donor molecule alone, there were no meaningful values for $\mathrm{a}_{1} / \mathrm{a}_{2}$ ratio since it is a single exponential decay. (Becker \& Hickle TCSPC FLIM board was integrated with Biorad Radiance2100 confocal/multiphoton microscopy [1]. CFPex-820 nm; CFPem-480/40 nm; YFPex-920 nm; YFPem540/30 nm; Nikon objective lens-60x W NA=1.4) 